\title{
Robust Verification Analysis
}

\author{
William Rider ${ }^{\mathrm{a}, 1, *}$, Walt Witkowski ${ }^{\mathrm{b}}$, James R. Kamm ${ }^{\mathrm{c}}$, Tim Wildey ${ }^{\mathrm{a}}$ \\ ${ }^{a}$ Sandia National Laboratories, Center for Computing Research, Albuquerque, NM \\ 87185, United States \\ ${ }^{b}$ Sandia National Laboratories, Verification and Validation, Uncertainty Quantification, \\ Credibility Processes Department, Engineering Sciences Center, Albuquerque, NM 87185, \\ United States \\ ${ }^{c}$ Los Alamos National Laboratory, Methods and Algorithms Group, Computational \\ Physics Division, Los Alamos, NM 87545, United States
}

\begin{abstract}
We introduce a new methodology for inferring the accuracy of computational simulations through the practice of solution verification. We demonstrate this methodology on examples from computational heat transfer, fluid dynamics and radiation transport. Our methodology is suited to both welland ill-behaved sequences of simulations. Our approach to the analysis of these sequences of simulations incorporates expert judgment into the process directly via a flexible optimization framework, and the application of robust statistics. The expert judgment is systematically applied as constraints to the analysis, and together with the robust statistics guards against overemphasis on anomalous analysis results. We have named our methodology Robust Verification.

Our methodology is based on utilizing multiple constrained optimization problems to solve the verification model in a manner that varies the analysis' underlying assumptions. Constraints applied in the analysis can include expert judgment regarding convergence rates (bounds and expectations) as well as bounding values for physical quantities (e.g., positivity of energy or density). This approach then produces a number of error models, which are then analyzed through robust statistical techniques (median instead of mean statistics).

\footnotetext{
${ }^{*}$ Corresponding author

Email address: wjrider@sandia.gov (William Rider)

${ }^{1}$ Sandia National Laboratories is a multi-program laboratory managed and operated by Sandia Corporation, a wholly owned subsidiary of Lockheed Martin Corporation, for the U.S. Department of Energy's National Nuclear Security Administration under contract DE-AC04-94AL85000.
} 
This provides self-contained, data and expert informed error estimation including uncertainties for both the solution itself and order of convergence. Our method produces high quality results for the well-behaved cases relatively consistent with existing practice. The methodology can also produce reliable results for ill-behaved circumstances predicated on appropriate expert judgment. We demonstrate the method and compare the results with standard approaches used for both code and solution verification on wellbehaved and ill-behaved simulations.

Keywords: Verification, error estimate, convergence, order-of-accuracy, solution verification, robust statistics

\section{The Role of Verification in Computational Modeling and Sim- ulation}

Verification is a process whereby the mathematical correctness and accuracy of methods and simulations can be determined. The foundation for verification is the theoretically determined properties of the numerical methods, which provide the expected behavior as the mesh spacing, time step size, or whatever discrete parameters control computational resolution, e.g., approximation order or solver tolerance. Typically, in verification, one systematically varies the discrete resolution and uses the results to either determine the rate of convergence, and/or the solution that is approached asymptotically. While other forms of error estimation can be applied, our work primarily applies to verification using varying resolution. The notion of convergence is central to all verification, which signifies that a limiting or asymptotic solution is approached as computational resources are increased $[19,20,16]$.

Verification is conducted in two modes, code verification and solution (or calculation) verification. Code verification is a well-defined process by which the correctness and accuracy of a software implementation of a numerical algorithm can be evaluated, usually by matching the rate of convergence with respect to discrete resolution to expectations from the numerical analysis of the method. Solution verification is a related, but distinct process where the discretization error is estimated in simulations of interest. Additionally solution verification provides an estimate of the limiting solution approached as resolution increases. In code verification the numerical solutions are compared with an exact solution while solution verification does not have an exact (or fiducial) solution. The class of solutions to be considered for code verification includes those defined by the method of manufactured solutions 
(MMS) as well. In code verification, the rate of convergence is paramount in importance, while in solution verification the numerical error estimate is the quantity of primary interest.

Most numerical methods used to obtain approximate numerical solutions of continuum models have a number of key properties. Among these characteristics is the order-of-accuracy (also called the rate of convergence), which is given by the exponent in the power law relating the numerical truncation error to the value of the discretization parameter. For example, if a method is first-order accurate the error should behave like, $C_{1} h+o(h)$ where $C_{1}$ is a constant, and $h$ is the discrete step size, and the notation " $o\left(h^{p}\right)$ " denotes terms that approach zero faster than $h^{p}$ as $h \rightarrow 0$. A second-order method will have an error that scales differently, $C_{2} h^{2}+o\left(h^{2}\right)$ and so on. The most common approach to code verification is to compare the theoretical rate of convergence of the numerical method to the observed rate produced by an implementation of that method, to gauge the correctness of the implementation with which the error is clearly defined in a computed solution. This is also predicated upon the solution itself having sufficient regularity to support the theoretical rate of convergence. In other words, the solution must have enough well-defined derivatives to allow a certain order of accuracy to be achieved.

In the following sections we first review the basic verification approach accepted broadly and used in practice. This is followed by a brief critique and the development of our method as an extension the basic approach. We then describe our improved approach that eliminates the necessity of explicitly introducing safety factors. We describe a circumstance where expert judgment is necessary in existing verification practice, and techniques to more automatically include it in the analysis. We then introduce our method that provides a set of estimates using an optimization framework that produce an estimate of uncertainty through robust statistical analysis of results. Each of the individual estimates can include expert judgment as constraints in the optimization solution. In effect, the safety factor is automatically computed based upon the data and any expert judgment included in the analysis. Our approach also produces an uncertainty estimate for the rate of convergence, which may be useful in interpreting the overall quality of the results. We finish the paper with a set of results focusing upon solution verification of ideal and more applied problems. We also include a brief demonstration of code verification. 


\section{Standard Methods for Verification Analysis}

In this section, we discuss the standard setting for convergence analysis in verification. The axiomatic premise of asymptotic convergence analysis is that the computed difference between the reference and computed solutions can be expanded in a power series in the (length) scale of the discretization. Taking the spatial mesh as the archetypical example, the ansatz for the error in a simulation using solution verification with a well-defined mesh length scale is taken to be

$$
\left\|A_{\text {exact }}-A_{k}\right\|_{q}=C_{0}+C_{1} h_{k}^{p}+o\left(h_{k}^{p}\right) .
$$

In this equation, $A_{\text {exact }}$ is the reference or exact solution, which for solution verification is computed on the most refined mesh, $A_{k}$ is the computed solution, $q$ refers to the space for the norm of the difference, $h_{k}$ is the measure of the mesh-cell size, $C_{0}$ is the zero-th order error, $C_{1}$ is the coefficient of the leading order error.For consistent numerical methods, $C_{0}$ should be identically zero; we assume this to be the case in the following discussion. For consistent methods, the exponent, $p$, of $h_{k}$ is the convergence rate $(p=1$ implies first-order convergence, $p=2$ implies second order convergence, etc.).

The error ansatz implies:

$$
\left\|A_{\text {exact }}-A_{k}\right\|_{q}=C_{0}+C_{1} h_{k}^{p}+\ldots
$$

Now consider the case where the exact solution, $A_{\text {exact }}$ is not available. Instead we assume that the solution on the refined mesh, $k$, is smaller in error than a coarser mesh, $k-1$. Let us further assume that we have computational results on a "fine" mesh $h_{k}$ (subscript $k$ ), where $0<h_{k}<h_{k-1}$. In this case, the error ansatz can be manipulated to give:

$$
\left\|A_{k}-A_{k-1}\right\|_{q}=C\left(h_{k}^{p}-h_{k-1}^{p}\right)+\ldots,
$$

dropping the subscript 1 from the constant, $C$. Given a set of solutions, $A_{k}$, this produces a system of nonlinear equations that may be solved through standard numerical methods. This solution then allows for the estimation of numerical error for a given mesh once $C$ and $p$ are known (assuming that $C_{0}$ is identically zero). For a limited number of cases (three systematically chosen grids) these equations may yield to analytic solution.

For quantities of interest (QoIs) or figures of merit, the above development can be utilized without resorting to error norms. The quantity, $\tilde{A}$, is defined without the use of a norm with the following related error model,

$$
A_{k}=\tilde{A}+C h_{k}^{p}+\ldots
$$


with the remainder of the development proceeding as above, if the approach toward $\tilde{A}$, the mesh converged solution, is monotonic. In the case where a solution is not monotonically approached, the above error model can still be utilized as long as the error in absolute terms is diminishing monotonically. This model has a similar form,

$$
|E|=C h_{k}^{p}+\ldots,
$$

where $E$ is the error, which is almost identical to the form utilized for code verification.

\section{Safety Factors in Verification}

One of the important aspects of solution verification is the safety factor used to relate the distance between a numerical solution and the estimate of the mesh converged solution to a numerical error estimate (or bound). As such, the safety factor plays a crucial role in providing the quantitative numerical uncertainty value. A safety factor is an established engineering approach to account for the uncertainty in calculations supporting safety, design and operation. Practically the safety factor should balance considerations of securely bounding potential values while minimizing the costs attendant in a too generous value. The safety factor usually carries a particular probabilistic assumption which provides a $95 \%$ confidence bound (or two standard deviations for a normal distribution).

Roache introduced the safety factor into solution verification $[19,20]$. The safety factor is used to produce a numerical error estimate,

$$
U_{\text {num }}=F_{s}\left|\tilde{A}-A_{N}\right|
$$

where $F_{s}$ is the safety factor and $A_{N}$ is the most resolved calculation (i.e., with the smallest discrete step size). These safety factors took a relatively simple form and utilized Roache's experience in computational fluid dynamics (CFD) to good effect in their determination. Based on considerations from Roache's expertise in CFD the value for the safety factor was given as $F_{s}=1.25$ for well-behaved, usual cases where the solution is convergent in an identifiably normal fashion, and the number of meshes used for verification is sufficient. In the case where an inadequate number of meshes (usually two for spatial mesh refinement and associated error estimation) are used, the safety factor is increased to a value of $F_{s}=3$ to account for various issues (and perhaps to penalize these situations to some extent). 
Eça and Hoekstra [6] propose heuristics by which to estimate the numerical uncertainty associated with fundamental behavior of a set of computed results. This work has continued and recently extended using a larger number of meshes combined with advanced regression techniques [7, 5]. This work increases the capacity of verification to attack more realistic problems with a higher degree of certainty and precision. These suggestions appear to be based on the assumption that the underlying numerical scheme has a theoretical convergence rate of two; however, for certain discretizations and for many multiphysics (and some single-physics) problems, the theoretical convergence rate is unity, for which the specific prescription of [6] should be modified (see for example $[15,1,2]$ ).

Oberkampf and Roy [16] have provided an approach to more automatically make decisions regarding the setting of Roache's safety factors. In this approach there is a threshold set using the computed convergence rate (assuming three grids) and the larger safety factor is triggered by a convergence rate that is too far from the asymptotic rate expected (10\%). We highlight the heuristic, but simple estimation associated with Roache's procedure as defined by Oberkampf and Roy [16]. The simplicity of this estimate is in contrast to the more elaborate procedure described later. For both procedures, the starting point is a regression given the results of the mesh refinement (or coarsening) procedure. This produces a mesh-converged result, $\tilde{A}$, and convergence rate, $p$. From these values, one obtains the basic scale for the error estimate,

$$
\delta_{\alpha}=\frac{A_{k}-A_{k-1}}{\sigma^{p}-1}=\tilde{A}-A_{k} .
$$

This value is processed with the resultant convergence rate to define a safety factor,

$$
U_{\text {num }}=F_{s}\left|\delta_{\alpha}\right|= \begin{cases}1.25\left|\delta_{\alpha}\right|, & \text { if }\left|p-p_{t h}\right| / p_{t h}<0.1 \\ 3\left|\delta_{\alpha}\right|, & \text { otherwise, }\end{cases}
$$

where $p_{t h}$ is the theoretical convergence rate. Finally, the grid convergence index (GCI) is the ratio of $U_{\text {num }} / \tilde{A}$ expressed as a percentage. Today this approach is also codified by several professional standards.

Another approach was the Correction Factor (CF) method of Stern et al. [24] Xing and Stern [25]. This produces a somewhat more complex closed form structure of the safety factor, with the form dependinh on the rate of convergence computed. The functional form of the safety factor was then determined empirically to capture the true error at the prescribed level of confidence. As we describe below, Xing and Stern come to a different conclusion regarding an approach that technically does meet the $95 \%$ confidence 
level empirically, albeit with respect to a specific ensemble of simulations. The applicability of their approach outside the area studied in deriving their formula remains unresolved at the time of publication. Xing and Stern [25] take a different, more complicated, but nevertheless still empirical approach. To evaluate the numerical uncertainty associated with these solution verification estimates, Xing and Stern performed a statistical analysis of 25 sets of computational data, covering a range of fluid, thermal, and structural simulations, to arrive at various parameters for their estimations of simulation uncertainty. The parameter values obtained by Xing and Stern provide computational uncertainty estimates,

$$
U_{\text {num }}=F_{s}\left|\delta_{\alpha}\right|= \begin{cases}(2.45-0.85 P)\left|\delta_{\alpha}\right|, & \text { if } 0<P<1, \\ (16.4 P-14.8)\left|\delta_{\alpha}\right|, & \text { if } P>1,\end{cases}
$$

where $P=p_{R E} / p_{t h}$, the ratio of the Richardson extrapolation based convergence rate $\left(p_{R E}\right)$ and the theoretical convergence rate $\left(p_{t h}\right)$, indicates whether the observed solution is asymptotic in nature. The numerical error magnitude comes from the Richardson extrapolation as described by Equation (7).

Our goal is to provide a technique to produce safety factors and numerical error estimates in a self-contained fashion where the only dependence is on the data available, the nature of calculations with metrics and measures of interest, and the expert knowledge of the numerical methods, algorithms and codes being used. The technique we describe does not produce a "safety factor" per se, but rather its outcome could be interpreted as a safety factor if desired. Instead we represent the associated uncertainty inherent in the data and its concomitant analysis in a self-contained framework.

\section{The Need for Expert Judgment in Verification}

Verification results often require intervention by an expert once the standard analysis is completed. When calculations are under-resolved, or generally poor quality in some fashion the results from verification analysis are unreliable. For example the rate of convergence may indicate a problem if it is either abnormally low (or even divergent), or too high. Sometimes quite simple reasoning determines the expected rate of convergence, but the situation is often more complex. For example, if the problem being simulated is insufficiently smooth, the rate of convergence may be limited by the regularity of the solution and not the numerical method. Often, an expert's judgment is necessary to apply this maxim properly. 
It is instructive to provide a concrete example of these issues. In conducting very expensive calculations it is a profitable approach to conduct relatively inexpensive coarse grid calculations first. If properly executed the coarse grid calculations can be used to estimate the level of effort necessary for and the efficacy of refined calculations. Of course we state the obvious proviso that the coarse grid solution must capture important aspects of the physics of the solution being simulated. Using solution verification the level of numerical error may be estimated, and the mesh resolution needed for a calculation of given accuracy can be estimated. These estimates can then be better assessed once the more expensive calculations are conducted.

In [21], the authors presented a sequence of calculations for the analysis of large eddy simulations of fluid flow and heat transfer in a standard nuclear reactor rod bundle. These calculations used Sandia National Labs code, Fuego, and the standard Smagorinsky subgrid model. A set of relatively coarse grids were generated to assess grid sensitivity and overall solution cost. These grids were necessarily unstructured and variable in mesh density because of the geometric complexity of grid spacers used in the rod bundles for structural integrity and turbulence generation for the purpose of enhanced heat transfer. These calculations were conducted and analyzed for the purpose of planning more extensive calculations on refined meshes.

For simplicity, we examined the overall computed pressure drop for three calculations. The mesh density was quite simply characterized by its volumetric average computed by the cube root of the number of computational element divided by the physical volume, $h=(V / N)^{\frac{1}{3}}$. We readily acknowledge that this is a quite crude measure, but each mesh is otherwise similarly generated with the same methodology in a mesh generation code. We then use a standard for estimating the mesh converged pressure drop, $\Delta P=\Delta P_{k}+C h_{k}^{p}$.

We are given three pieces of data and three unknowns, a seemingly ideal circumstance. The solutions are consistent with a monotonically behaved solution with ordered pairs $(h, \Delta P)=(1.43,31.8 \mathrm{kPa}),(1.16,24.6 \mathrm{kPa}),(1.00,24.4 \mathrm{kPa})$. The mesh is the ratio of the meshes scale to that of the third and finest mesh. The calculations are conducted on meshes with $0.66,1.22$, and 1.93 million computational elements. This system of equations can be exactly solved and provides $24.3 \mathrm{kPa}=\Delta P_{k}+26.6 h_{k}^{15.84}$. This provides an estimate of numerical error using standard GCI methods of approximately $0.08 \mathrm{kPa}$ $\left(F_{s}=3\right)$. Xing and Stern's approach provides an uncertainty estimate of 1.6 $\mathrm{kPa}$ by virtue of the unusually high rate of convergence. Both imply that the calculations are already relatively converged. 
The expert judgment comes into play immediately, the very high convergence rate is completely unrealistic and calls the sequence of calculations into question. The code could be expected to give second-order results for smooth flows at best, and for a rough turbulent flow on a coarse mesh, first-order may be a more reasonable expectation. In many respects the large eddy simulation can be viewed as being quite similar to an early shock capturing method with an artificial viscosity [23], which provides additional support for first-order accuracy expected for those methods when underresolved. In the case of a Smagorinsky subgrid model, the solution method used here may be viewed as a base numerical method with the addition of an explicit artificial viscosity term. In addition, on purely physical terms the meshes are poorly resolved and almost certainly quite under-resolved, thus the error estimate simply is not credible. Given this suspicion we might ask, are these calculations a waste of time and effort? Or can some useful information be gleaned through a more refined analysis?

Constraining the fit of the data to the error model to give a convergence rate no larger than two immediately provides a far more reasonable analysis. The constrained fit is $15.7 \mathrm{kPa}=\Delta P(h)+7650 h^{2.00}$. Now the GCI procedure gives a numerical error of $25.9 \mathrm{kPa}$, while Xing and Stern give $18.7 \mathrm{kPa}$. Both results communicate the lack of convergence and the acknowledged unreliability of the sequence of calculations.

This turns out to be a useful test for our new robust verification methodology as well. We suggest that expert judgment be engaged prior to any analysis, and our goal is to provide a means for this judgment to be included in the analysis. The constrained optimization used in the follow-on to the initial analysis is simply the barest application of this principle. Even this minor augmentation provides substantial value. As we will elaborate upon later in the paper, the expert judgment on the reasonable convergence rates can be applied as constraints to the optimization process used to estimate error. We also change the norm for optimality of fit in the optimization procedure, and allow data to be excluded. We find that this combination of steps provides more plausible results, and, in retrospect, reliable estimates. At the beginning of Section 7 we will present the impact of our procedure on the analysis of this dataset.

The process we define next produces a result that performs similarly and provides useful results whether one has two solutions, three solutions, or many solutions. The other benefit is a natural and explicit inclusion of expert judgment in verification analysis from the outset of the analysis rather than an ex post facto check on the validity of the results or implicit expert judgement associated with algorithmic choices. If the analysis proceeds in 
a fashion that requires no expert intervention (i.e., the constraints are not active), the expert's influence on the results is minimal.

\section{A Robust Approach Using Constrained Optimization}

The starting point for verification analysis is the definition of a postulated model for the numerical error. The standard model is a power law, which we continue to study in modified form,

$$
A_{k}=\tilde{A}+C h_{k}^{p}
$$

a truncated version of Equation (4) where $A_{k}$ is the value computed on the $k$-th mesh, $\tilde{A}$ is the (estimate of the) mesh converged solution, $C$ is a proportionality constant, $h_{k}$ is the mesh length scale (e.g., cell size in 1D), and $p$ is the convergence rate. Often verification (in particular, code verification) focuses on the convergence rate, $p$, as the key result and its congruence with theoretical expectations, $p_{t h}$. In solution verification, the focus can be expanded to the overall error term, $C h_{k}^{p}$, with specific application to error estimation.

We repeat the important point that the theoretical convergence rate, $p_{t h}$, is dependent not only upon the method used for computations, but also upon the nature of the solution itself, the quantity whose convergence is being analyzed, and the metric being considered. For example, a second-order method can be used to compute a result, but the presence of a discontinuity can render the solution only first-order convergent at best [15].

To help illuminate the significance of this choice, we can examine some basic properties of the least squares curve fit. The least squares approach is directly associated with normal (Gaussian) statistical assumptions regarding the errors in the parameter values [3]. Specifically, the nonlinear least squares fit is optimal if the errors in the parameters are normally distributed about the optimal values. The least squares formulation has distinct virtues for the linear regression problem, because the solution is rendered linear by the minimization of fit residual in the $L_{2}$ norm. This property is lost when moving to nonlinear models, such as those we will utilize here. Consequently, we lose little in moving to a more general formalism for the fit through optimization.

This allows us to pose the minimization functional more generally as well as access solution techniques more robust than the general nonlinear regression methods allow. In particular, Tihkonov regularized least squares or LASSO regression can all be easily defined along with a reliable solution to 
the $L_{1}, L_{1 / 2}, L_{2}, L_{4}, L_{8}$ and $L_{\infty}$ optimization problems. In our experience, the solution methods used for the minimization are more flexible, reliable, and robust than those typically used for regression. Additionally, significant additional flexibility was gained by using optimization instead of regression.

The field of robust statistics has been developed to reduce the sensitivity of regression (or optimization) procedures to outliers or scatter in the data in a given data set. The simplest robust regression approach is to minimize the $L_{1}$ norm of the residual. In distinction to the least-squares approach mentioned above, the $L_{1}$ regression has a different statistical connection. For $L_{1}$ regression, the fit is optimal if the errors are distributed by Laplace's (double-exponential) distribution[3]. The double-exponential distribution is sharply peaked at the mean and has longer tails than the normal distribution. Solving the $L_{1}$ problem also naturally removes outliers from the data. At the other end of spectrum is the minimization of the $L_{\infty}$ norm of the residuals (also known as Chebyshev or minimax approximations). Unlike minimization of the $L_{1}$ norm, $L_{\infty}$-based regression is minimally robust because it can be greatly influenced by outliers; nevertheless, this form of regression is indeed optimal if the errors are distributed uniformly.

We contend that such sensitivity is not an appropriate characteristic for a "best estimate" of the result. We make this assertion based on our experience that apparent outliers in the results of numerical calculations of computational science and engineering are far from unknown. To help address this issue, we choose instead the median of our estimates as the measure of central tendency. Unlike the mean, the median of a data set is substantially more robust to outliers [9]. The variation in the data can likewise be measured by the median deviation (analogous to the standard deviation), which is the median of the deviation from the median across the ensemble.

Finally, we can provide an improvement in the model fit via the application of weighting the data. We have the prior expectation that the results computed on finer grids (i.e., with smaller mesh spacing) are "better". This presumption is essentially a restatement of our belief, ultimately, of convergence under mesh refinement. To reflect this assumption quantitatively, the data can be weighted inversely proportional to the mesh spacing (i.e., by $\left.1 / h_{k}\right)^{2}$.

Another novel element of our approach it is the ability to examine the

\footnotetext{
${ }^{2}$ Of course, this weighting could be modified to be inversely proportional to the mesh spacing to some positive power, i.e., $1 / h_{k}^{q}$, where $q>0$.
} 
results in a manner that does not assume the symmetry of the estimates. The primary analysis produces estimates of the mesh converged result, $\tilde{A}$, which should not necessarily be symmetric about the median, but rather potentially have a bias. To accomplish this analysis we first compute the median of $\tilde{A}$ and then divide the list of estimates into two lists of estimates: those less than the median value and those greater. We difference these values from the median $(\tilde{A})$ for each element of these sets and then compute the median deviation for each list. These values are signed, and provide an estimate of the negative or positive bias in the estimate of numerical error.

\section{An Optimization Based Solution Verification Procedure}

These considerations have been incorporated into a procedure for producing an error estimate that naturally provides uncertainty in both the magnitude of the error and the rate of convergence. In addition, the procedure provides a conduit to include expert judgment into the estimates naturally. The procedure is self-consistent whether the number of calculations supports an under-, fully- or over-determined solution to Equation (10).

The basic procedural approach to producing best-estimates of numerical error as follows.

1. Use an analysis of the numerical method applied and the problem solved to establish an expected theoretical rate of convergence with lower and upper bounds for the convergence rate, $p_{\text {lower }} \leq p \leq p_{\text {upper }}$. For the more complex error ansatz with two discrete variables, bounds should be entered for all variables. In addition, the basic nature of the solution can be encoded as a constraint (such as positive definiteness, or more specific upper or lower bounds, $\tilde{A}_{\text {lower }} \leq \tilde{A} \leq \tilde{A}_{\text {upper }}$ ). This is the primary conduit for expert judgment to influence the results.

2. Screen the data for its basic character (i.e., whether the convergence is monotonically convergent, convergent, or divergent). For divergent datasets, the optimizations will not necessarily yield consistently improved results, although the workflow can be completed without issue. This typically shows itself as the procedure activating one of the constraints discussed in step 1 in most solutions for the Equation (10).

(a) Choose a data set based from the finest to coarsest mesh values $S_{1}=\left[\left(h_{N-1}, A_{N-1}\right),\left(h_{N}, A_{N}\right)\right], j=1$. Each data set in this simple approach is $S_{j}=\left[\left(h_{N-j}, A_{N-j}\right), \ldots,\left(h_{N}, A_{N}\right)\right]$. The mesh is characterized by a single value $h_{k}$, which is a representative length scale for the $k$ th mesh. Work through the data points 
starting with the finest resolution, adding additional (coarser) data points on each pass through and producing new optimization fits for each set of data. This aspect of the procedure is predicated upon the (typically justified) assumption that finer grids should produce more accurate results. Thus, for each part of the full data set, one obtains a set of optimizations, with the results biased toward the finer grids. Alternative approaches to consider are similar to the jack-knife procedure where points are systematically left out, or the bootstrapping procedure where the full data set is re-sampled to improve statistical properties.

(b) Using the subset of the data, $S_{j}$, produce the following steps.

(c) Using the data pairs $\left(h_{k}, A_{k}\right)$ produce a set of constrained optimizations using several techniques $L_{1}, L_{2}$, and $L_{\infty}$. In addition, we use $L_{4}, L_{8}$, Tikhonov, LASSO, as well as weighted variants of each using $\left(1 / h_{k}\right)$. Each of these problems can be computed using a constrained minimization statement of the problem,

$$
\begin{gathered}
\arg \min _{k}\left\|A_{k}-\tilde{A}-C h_{k}^{p}\right\|_{\beta} \\
\text { subject to } p_{\text {lower }} \leq p \leq p_{\text {upper }}, \tilde{A}_{\text {lower }} \leq \tilde{A} \leq \tilde{A}_{\text {upper }} .
\end{gathered}
$$

where $\beta$ is the appropriate norm to minimize in. We also solve a set of optimization problems where the rate of convergence is defined by the constraints, $p_{\text {lower }}, p_{t h}$, and $p_{\text {upper }}$,

$$
\begin{array}{r}
\arg \min _{k}\left\|A_{k}-\tilde{A}-C h_{k}^{p_{\text {th }}}\right\|_{2} \\
\arg \min _{k}\left\|A_{k}-\tilde{A}-C h_{k}^{p_{\text {lower }}}\right\|_{2} \\
\arg \min _{k}\left\|A_{k}-\tilde{A}-C h_{k}^{p_{\text {upper }}}\right\|_{2} .
\end{array}
$$

In the case where either data is sparse (the under-determined case), or for very well-behaved data where most results produce $p \approx p_{t h}$ the use of the lower and upper bounds will produce the lower and upper bounds on $\tilde{A}$. In addition we could envision solving problems for the constrained values for $\tilde{A}$

$$
\begin{gathered}
\arg \min _{k}\left\|A_{k}-\tilde{A}_{\text {lower }}-C h_{k}^{p}\right\|_{\beta} \\
\arg \min _{k}\left\|A_{k}-\tilde{A}_{\text {upper }}-C h_{k}^{p}\right\|_{\beta},
\end{gathered}
$$

but we have not yet pursued this option. This would have the effect of producing bounding values for $p$. For sufficient amounts 
of data with well-behaved solutions these bounding values wil not impact the error estimation because of the impact of using median statistics in making the final estimates will remove these solutions from consideration.

(d) Examine the results to see whether the computed estimates of $p$ match either the lower or upper bound. Similarly examine the results to see whether the estimates of $\tilde{A}$ match the lower or upper bound. This is a warning sign that probably precludes the completion of a "best estimate" of $\tilde{A}$. If the estimates do not activate the constraints consistently they are interpreted as valid and provide valuable information on the inferred values for $p$ and $\tilde{A}$. Variations in these estimates provide uncertainty based on the data. ${ }^{3}$

(e) Return to step 2a until the data are exhausted.

3. With the data exhausted and a collection of optimizations (using the index $i=1, \ldots M)$, find the median ${ }^{4}$ of $\tilde{A}, \tilde{A}_{\text {median }}$, the median deviation, $\Sigma_{\text {median }}=$ median $\left\|\tilde{A}_{i}-\tilde{A}_{\text {median }}\right\|$. The estimate of the mesh converged solution is $\tilde{A}_{\text {median }} \pm 3 \Sigma_{\text {median }}$. The reasoning for choosing $3 \Sigma_{\text {median }}$ is to provide a bound analogous to the $95 \%$ confidence interval sought with other solution verification procedures. For a normal distribution two standard deviations is equivalent to three median deviations $[4,22,13]$.

4. Conduct the same analysis for rate of convergence for all the optimizations to provide the estimated rate of convergence and its uncertainty, $p_{\text {median }} \pm 3 \rho_{\text {median }}$ where $\rho_{\text {median }}=$ median $\left\|p_{\text {median }}-p_{j}\right\|$.

5 . Conduct the asymmetric analysis of the results by separating a sorted list of the $\tilde{A}$ into two equal lists, one with elements less than $\tilde{A}_{\text {median }}$ and the other greater than $\tilde{A}_{\text {median }}$. Compute the median of each of these sets and subtract $\tilde{A}_{\text {median }}$, which provides a negative and positive bias, $3 \Sigma-$ and $3 \Sigma+$, in $\tilde{A}_{\text {median }}$. The same can be completed for the rate of convergence.

\footnotetext{
${ }^{3}$ The actual optimization algorithms used in the analysis are found in the software Mathematica. The default option of Nelder-Mead is used for the fixed convergence rate solution, simulated annealing is used for most cases except for the difficult, $L_{1}$ and $L_{1 / 2}$ optimizations where a random search is applied. In all cases the constraints are implemented using a penalty methodology.

${ }^{4}$ The median is the number that separates an ordered list of values into two equal length lists one less than the median and the other greater than the median. Should the length of the list be an even number, the median is the arithmetic average of the two values meeting the above definition.
} 
6. For all results, one can produce a "GCI-like" result in terms of percentage taking the additional numerical uncertainty into account (and converting it to a percentage). In addition one may compute an effective safety factor by taking the difference between the finest mesh solution and adding the error uncertainty,

$$
U_{\text {num }}=\left|\tilde{A}_{\text {median }}-A_{N}\right|+3 \Sigma_{\text {median }},
$$

and

$$
F_{s}=\frac{\left|\tilde{A}_{\text {median }}-A_{N}\right|+3 \Sigma_{\text {median }}}{\left|\tilde{A}_{\text {median }}-A_{N}\right|} .
$$

Should the data be convergent, but oscillatory rather than monotonic, a small change can be used in the procedure. The basic process simply looks at the absolute change in the solution under changes in resolution rather than the signed solution values. No other substantive change is made in the overall procedure.

If the absolute value of the error is monotonically convergent (this includes the monotonically convergent case), we can also solve the related optimization problem:

1. Compute the absolute difference between the solutions at adjoining meshes, $\left(h_{k}, h_{k-1},\left|A_{k}-A_{k-1}\right|\right)$ (define $\Delta A_{k, k-1}:=\left|A_{k}-A_{k-1}\right|$.

(a) Produce a set of optimizations as before using the same collection of error norms,

$$
\begin{aligned}
& \arg \min _{k}\left\|\Delta A_{k, k-1}-C h_{k}^{p}+C h_{k-1}^{p}\right\|_{\beta} \\
& \text { subject to } p_{\text {lower }} \leq p \leq p_{\text {upper }}, C>0 .
\end{aligned}
$$

We note that this particular approach can also be used to conduct code verification with several small changes, $\left(h_{k},\left|A_{\text {exact }}-A_{k}\right|\right)$,

$$
\begin{gathered}
\arg \min _{k}\left\|E-C h_{k}^{p}\right\|_{\beta} \\
C>0 .
\end{gathered}
$$

(b) Screen the results of the optimizations for anomalous behavior in convergence rates. Pass through the data from fine to coarse as before.

(c) For the best estimate of error, use the median of the error model, $C h_{n}^{p}$ optimal fits evaluated at $h_{n}$, where $n$ is the finest grid available. This is the best estimate of the error bar. 
(d) If an upper bound on error is desired, find the $\max \left(C h_{n}^{p}\right)$ for an estimate on the finest grid.

(e) As before pass through the data until it is exhausted.

2. If the errors diverge, compute the rate of divergence and exit .

3. Apply the median statistical analysis as before to provide best estimates with uncertainty.

It is worthwhile to make the following comments on the procedure. Expert judgment is added to the process in several key places: the determination of the expected convergence, the screening of the data (with potential rejection of anomalous solutions, and the screening of the optimization results). The use of robust statistics can provide some relief from this step, but expert opinion remains a necessary element in this activity, but is introduced in a structured manner. Finally, the procedures eliminate the use of an explicit safety factor. Now the diversity of estimates provides the function of the safety factor via the reduction of the estimates via robust statistics.

\section{Results}

Before presenting new results we will briefly return to the example of large eddy simulation presented in Section 4 as motivation for the need for expert judgment in verification. Using the procedure defined in Section 6 we achieve more reasonable results. The mesh converged estimate of the pressure drop is $16.0 \mathrm{kPa} \pm 3.9 \mathrm{kPa}$ using the constraint of $0.5 \leq p \leq 2.0$. These estimates would indicate that to achieve a numerical error of one $\mathrm{kPa}$ or less would require a mesh of approximately a billion elements. The rate of convergence has been constrained to be reasonable given expert judgment and the magnitude of numerical error is estimated to be quite large. The estimate of the rate of convergence is $p=1.14 \pm 0.60$. Furthermore the rate of convergence itself has an uncertainty. It is notable that this is consistent with far more refined calculations conducted with a 5.8 and 12.5 million element meshes similarly generated to the meshes discussed above, $(h, \Delta P)=(0.56,22.0 \mathrm{kPa}),(0.43,20.8 \mathrm{kPa})$. These calculations were conducted with a different computer code and method, but were otherwise similar $[17,14,18]$. The estimate of the mesh converged pressure drop there is $16.3 \mathrm{kPa} \pm 7.0 \mathrm{kPa}$. These results provide confirmation that the estimates

\footnotetext{
${ }^{4}$ For under-determined ( 2 grid) cases, this cannot be explicitly determined. We further note that the error examination has been excluded from this study for brevity.
} 
on the extremely coarse meshes are useful using our new procedure. This strongly suggests that the new approach provides a valuable contribution to our computational simulation capability.

\subsection{Simple Example Using a 1st Order Integration of an ODE}

To clearly demonstrate these techniques we apply both the code and solution verification methodology to a simple problem as an example. To this end, we will solve a linear ODE, $\frac{d A}{d t}=-A, \quad A(0)=1$, for the solution at time $t=2$, using the first-order accurate forward Euler method, $A^{n+1}=A^{n}-h A^{n}$. The results are given in Table 1 for the analysis below. The analytical solution is $A(2)=0.135335 \ldots$... By utilizing the exact solution, we demonstrate our code verification methodology, and by ignoring the exact solution we demonstrate (and quantify the accuracy of) the solution verification techniques.

Table 1: First order forward Euler solution of an ODE for varying time step sizes. The exact solution at $t=2$ is 0.135335 for reference.

\begin{tabular}{lll} 
Time Step Size & Solution at $t=2$ & Exact Error at $t=2$ \\
\hline 0.4 & 0.077760 & 0.05758 \\
0.25 & 0.10011 & 0.03522 \\
0.2 & 0.10737 & 0.02796 \\
0.1 & 0.12157 & 0.01376 \\
0.08 & 0.12436 & 0.01097 \\
0.05 & 0.12851 & 0.00682 \\
0.04 & 0.12989 & 0.00546 \\
0.02 & 0.13261 & 0.00273 \\
0.01 & 0.13398 & 0.00136 \\
0.008 & 0.13425 & 0.00108 \\
0.005 & 0.13466 & 0.00068 \\
0.004 & 0.13479 & 0.00054 \\
\hline
\end{tabular}

\subsubsection{Code Verification}

The code verification results can be computed using the standard techniques with a single linear regression (including a standard deviation computed using Gaussian statistics). In this case, the data in Table 1 gives a 
convergence rate of $1.03150 \pm 0.0029816 .{ }^{5}$ Our new methodology provides very nearly the same result, but, by applying a range of regressions on subsets of the data, uncertainty in the convergence rate is also estimated, with the result: $1.00436 \pm 0.009538$, based on 209 different fits. Our procedure provides a result that focuses upon the fine grid results and provides great confidence that the integrator is implemented correctly. In this case the expected theoretical convergence rate lies well within the computed uncertainty from the procedure. The same sequence of actions can be applied while ignoring the exact solution to produce numerical error estimates.

\subsubsection{Solution Verification with The Full Data Set}

In order to judge results for more limited data we first analyze the full data set given in Table 1 . Here, we have constrained the convergence rate to be in the interval $0.5 \leq p \leq 1.5$ in the analysis. The new procedure is accurate for this well-behaved case with a great deal of data. With this amount of data the constraints have little or no influence on the results, which is distinctly different than the data poor cases explored later. We will compare this with the more traditional approaches. In contrast the new procedure provides a more self-contained error estimate with uncertainty together with a convergence rate and uncertainty. Figures 1 and 2 provide a snapshot picture of the sampling used by our procedure. On the other hand, our bounding error estimate is $0.0005351 \pm 0.0000154$, which is extremely accurate given the exact value for the error given in Table 1.

This is an almost ideal case that should be a straightforward for almost any methodology. Our new procedure gives the following estimate for $A(2.0), A_{k}=0.1353 \pm 0.00006936+C h_{k}^{1.0226 \pm 0.04881}$ using 209 models (11 subsets of the data with 19 models each). This corresponds to a safety factor of $F_{s}=1.133$, for $\Delta A=0.0005884$. The standard least squares fit is $A_{k}=0.1352-0.14862 h_{k}^{1.0388}$. The GCI approach gives a numerical uncertainty of $\Delta A=0.0005179, F_{s}=1.25$. Stern's approach gives an uncertainty of $\Delta A=0.0004323, F_{s}=1.043$. Stern and Xing's approach provides $\Delta A=0.0009263, F_{s}=2.236$ as the numerical uncertainty.

One might be tempted to compute fewer models with our procedure and only pass through the data once. This provides an estimate based on 19 models using the full data set $A_{k}=0.1352 \pm 0.0001397+C h_{k}^{1.0372 \pm 0.01031}$.

\footnotetext{
${ }^{5}$ Part of the selection of the step sizes is intended to be non-ideal. For code verification the standard is step doubling (or halving), but more difficulty in verification occurs for more general changes in step size. We are interested in determining the performance of our method under less than ideal circumstances.
} 


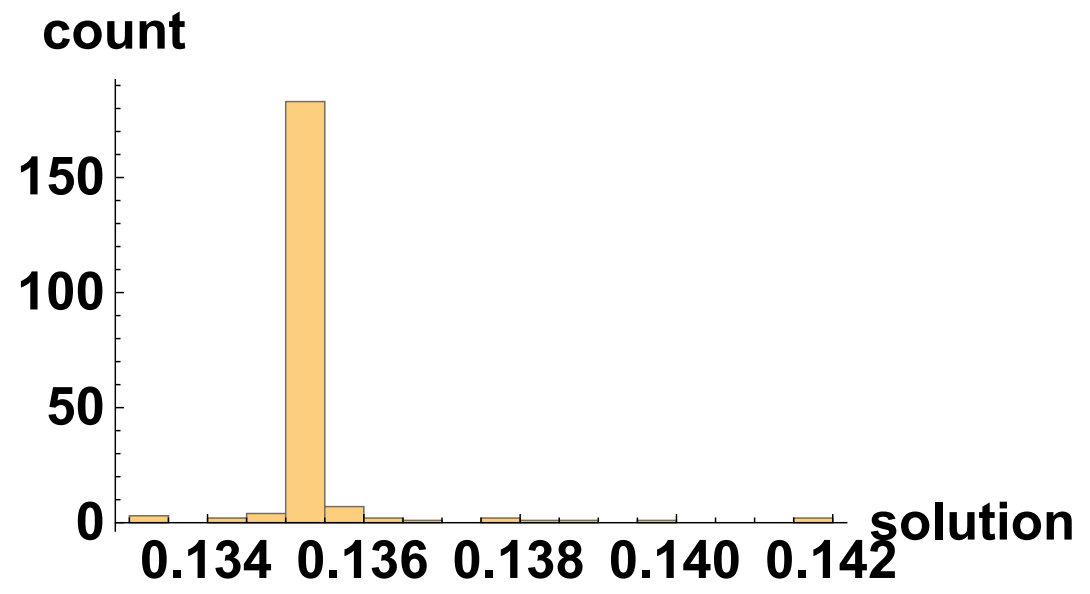

Figure 1: Calculated histogram (i.e., the effective PDF) of the estimated mesh converged result $\tilde{A}$ for the ideal case in the ODE example verification example. The histogram is nonsymmetric and biased toward values less than the peak. The exact solution is contained in the bin associated with the peak of the PDF. This bias is well described by the difference in the computed median deviation values $\Sigma-$ and $\Sigma+$, where the negative deviation is ten times larger than the positive deviation.

The error estimate from the full estimation procedure gives an estimate of the solution that is much closer to the exact solution. For the full procedure, the actual numerical error using the exact solution is $\Delta A=0.00002589$. The single pass solution gives $\Delta A=0.00008311$, which is a bit more than three times larger. The standard least squares fit to the data gives $\Delta A=$ 0.0001274 or another one and a half times larger again. In a sense this example illustrates the level of benefit associated with our full procedure under nearly ideal circumstances.

\subsubsection{Solution Verification with Two Points}

Because the methodology was developed to specifically provide verification analysis for data sets that are far from ideal, we will produce the solution verification analysis using two, three, and four non-ideal coarsely resolved data points. These points will be chosen from the largest step sizes in the data set; in the case of two points, $(0.40,0.077760)$, and $(0.25,0.10011)$. Normally this case resists analysis, and one would simply difference these two solutions and multiply by a safety factor $\left(F_{s}=3, U_{\text {num }}=0.06705\right)$. Instead we will use our procedure to provide a refined error estimate including a mesh converged solution and rate of convergence plus uncertainty. 


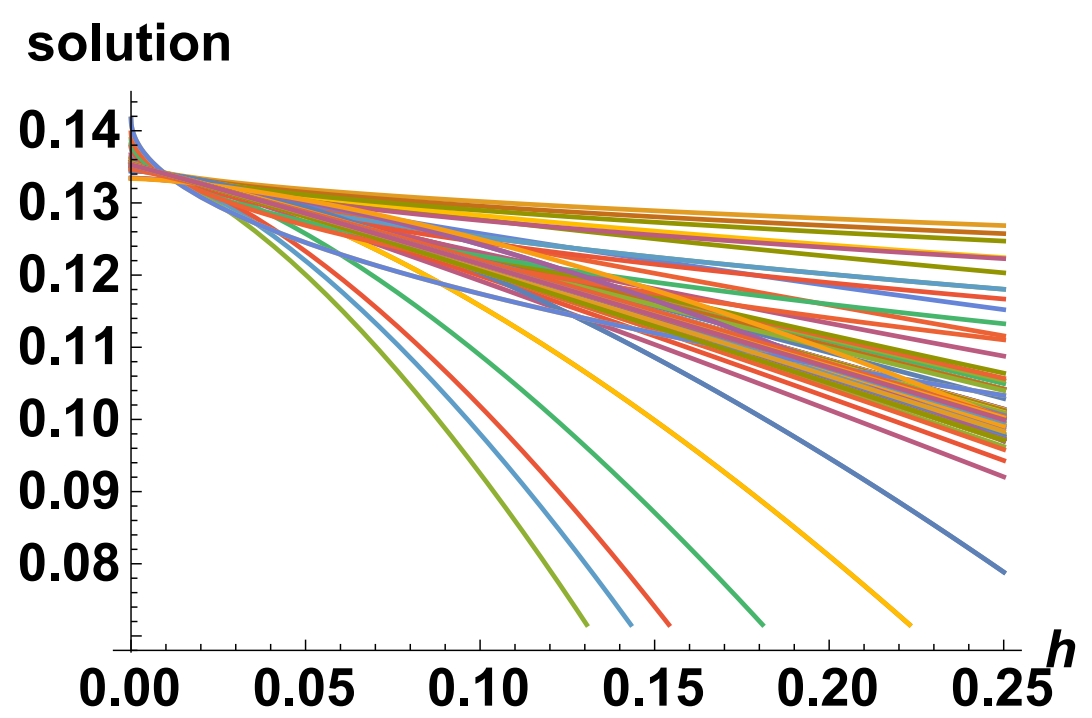

Figure 2: The ensemble of the 209 different solution models derived for the ODE case. Most of the models lie at the upper part of the plot, which strongly influences the estimates and uncertainty. The corresponding histogram (i.e., the effective PDF) is definitely not Gaussian in shape, as shown in Fig. 1. The values in the PDF are the quantities shown on the ordinate of this plot, i.e., the values at $h(\Delta t)=0$.

We make particular note that the two data point case is quite strongly influenced by the expert judgment. To demonstrate this point we can provide results from two choices representing tighter and looser constraints on the rate of convergence. The tighter case uses $0.75 \leq p \leq 1.25$ while the looser case uses $0.5 \leq p \leq 1.5$. For the tighter constraint the results using the new procedure is $0.1356 \pm 0.0088=A_{k}+C h_{k}^{1.0388 \pm 0.1318}$. The looser constraint gives a less pleasing result as might be expected $0.1245 \pm 0.0050=A_{k}+C h_{k}^{1.3962 \pm 0.1207}$. Neither the solution nor the rate of convergence is consistent with the finer stepsize calculations will produce. Each procedure is using 19 models in the error estimation. This produces an effective safety factor of $F_{s}=1.255$ and $F_{s}=1.164$, respectively. Clearly the expert judgment has a large impact on the result. This may not be satisfactory to many, but the alternative is to accept a result that depends upon expert judgment to much the same degree. If the constraints are inappropriately chosen, the results will suffer accordingly. For example, a large range of allowed convergence rates will result in a large uncertainty, conversely a tight convergence rate will produce a small variation. If the true 

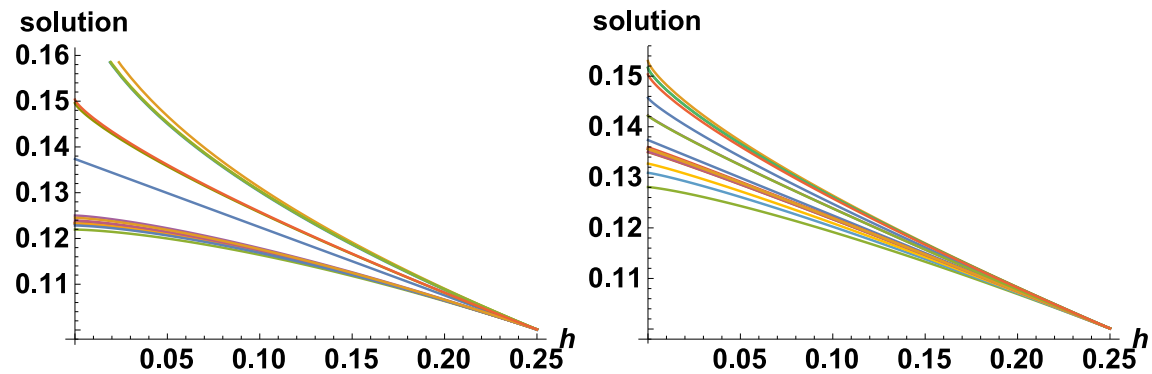

Figure 3: The sets of 19 models for the two coarse step size solutions of the first order ODE solution. The left plot shows the relatively loose constraint on the convergence rate, $0.5 \leq p \leq 1.5$, and the right plot shows the tigther constraint, $0.75 \leq p \leq 1.25$. The tighter constraint produces far smaller error and a more accurate estimate of the converged solution.

convergence rate is not contained in the allowed values, the results will not be reliable.

\subsubsection{Solution Verification with Three Points}

We now add a third data point $(0.20,0.10737)$ and compute the error estimates again. With a third data point the standard error model can be solved exactly. As one might expect the result is similar to the simple solution to the data using a standard fit because constraints are not activated by the data. The standard GCI approach gives $0.1343=A_{k}+0.1508 h_{k}^{1.0714}$ with $\Delta A=0.0336$. The tighter or looser constraints have a relatively small influence on the results. For the looser constraint we compute $0.1343 \pm$ $0.0043=A_{k}+C h_{k}^{1.0716 \pm 0.0979}$ and a $F_{s}=1.16$. The tighter constraint primarily produces a slightly smaller safety factor. In this case the procedure developed 38 different models for the estimates.

\subsubsection{Solution Verification with Four Points}

We now add a fourth data point $(0.10,0.12157)$ and compute the error estimates again. With a third data point the standard error model is now overdetermined. The standard GCI approach gives $0.1346=A_{k}+0.1504 h_{k}^{1.0619}$ and $\Delta A=0.0163$ in this case. The tighter or looser constraints have a smaller influence on the results than for the three data points. For the looser constraint we compute $0.1348 \pm 0.0016=A_{k}+C h_{k}^{1.0530 \pm 0.0499}$ and a $F_{s}=1.12$. As might be expected, more data means better solutions along with smaller safety factors and less influence of expert judgment. 
All of these results are summarized in Table 2 to allow ease of comparison.

Table 2: A summary of the verification results for the simple ODE case. All of the robust verification results use the loose constraint on the convergence rate, $0.5 \leq p \leq 1.5$ unless otherwise stated.

\begin{tabular}{lllll}
\hline Method & Number of Points & $\tilde{A}$ & $p$ & $F_{s}$ \\
\hline GCI & 2 & $\mathrm{~N} / \mathrm{A}$ & $\mathrm{N} / \mathrm{A}$ & 3.0 \\
Robust & 2 & $0.1245 \pm 0.0050$ & $1.3962 \pm 0.1207$ & 1.255 \\
Robust & 2 & $0.1356 \pm 0.0088$ & $1.0388 \pm 0.1318$ & 1.164 \\
$0.75 \leq p \leq 1.25$ & & & & \\
\hline GCI & 3 & 0.1343 & 1.0714 & 1.25 \\
Robust & 3 & $0.1343 \pm 0.0043$ & $1.0716 \pm 0.0979$ & 1.16 \\
\hline GCI & 4 & 0.1346 & 1.0619 & 1.25 \\
Robust & 4 & $0.1348 \pm 0.0016$ & $1.0530 \pm 0.0499$ & 1.12 \\
\hline GCI & 11 & 0.1352 & 1.0388 & 1.25 \\
Robust & 11 & $0.1353 \pm 0.00006936$ & $1.0226 \pm 0.04881$ & 1.133 \\
\hline
\end{tabular}

\subsection{Heat Transfer In a Simple Domain}

In this section, we solve the transient heat-equation

$$
\begin{cases}\frac{\partial T}{\partial t}-\nabla \cdot(K \nabla T)=f(x, t), & x \in \Omega, t>0, \\ T=g, & x \in \partial \Omega, t>0, . \\ T=T_{0}, & x \in \Omega, t=0,\end{cases}
$$

on an L-shaped domain (see Fig. 4). The diffusion coefficient in all examples is piecewise constant over subsets of the domain. We also allow homogeneous Neumann boundary conditions on a subset of the boundary. We numerically approximate the solution to these partial differential equations using a standard continuous Galerkin finite element method on a quasiuniform triangulation of the domain. In general, we use $h$ to denote the representative length scale of an element. If the mesh is uniform, then $h$ is constant throughout the domain. Otherwise, we define $h$ locally on each element. Triangular elements were chosen to allow for adaptive mesh refinement without incorporating hanging-node constraints. The polynomial 
degree is taken to be between 1 and 3 with the appropriate quadrature rule chosen to ensure that the spatial quadrature error is not significant. For the purposes of error estimation and solution verification the length scale of the smallest element will be used. This is a common expectation made by users of adaptive computations where an adaptive calculation is expected to produce results equivalent to a mesh where the entire domain is discretized at the smaller $h$. A second-order BDF method is used to integrate forward in time. In all of the time-dependent simulations the time step is selected so that the time discretization error is significantly smaller than the spatial discretization error. This "sufficiently small" time step was obtained by solving the problem on the finest spatial discretization with a sequence of decreasing time steps. In general, it is certainly possible to include the time discretization error into the analysis, but such an analysis is beyond the scope of this study.

The diffusion coefficient is given by

$$
K= \begin{cases}1, & (x, y) \in(0,1) \times(-1,0) \\ 0.01, & (x, y) \in(0,1) \times(0,1) \\ 1, & (x, y) \in(-1,0) \times(0,1)\end{cases}
$$

The region with the lowest diffusivity is $(0,1) \times(0,1)$, and the solution varies the most in this region as indicated in Fig. 5 . In general, discontinuous diffusion coefficients reduce the regularity of the solution from $h^{2}$ (or higher) to $h^{3 / 2}$ although the solution may be smooth away from the discontinuity.

We start from a homogeneous initial condition and evolve the solution in time. Our objective is to compare the time history of two quantities of interest and to define a race condition as the QoI. The race condition is the difference in time for two different locations to achieve a given set of temperatures. We consider the average temperature of the two boxes shown in Fig. 4.

The first box (Box 1 in Figure 4) heats up relatively quickly and reaches a steady state while the second box (Box 2 in Figure 4) heats up much more slowly and does not reach a steady state by the end of the simulation. Our quantity of interest is the time at which the temperature of the second box matches the temperature of the first box. This occurs at approximately $t=18$ on a relatively coarse spatial mesh. Our putative reference solution is a uniformly refined solution at a very fine mesh, which is used as a "benchmark". For problems exhibiting local singularities, as is often the case for problems with re-entrant corners, the spatial discretization error is usually dominant. 


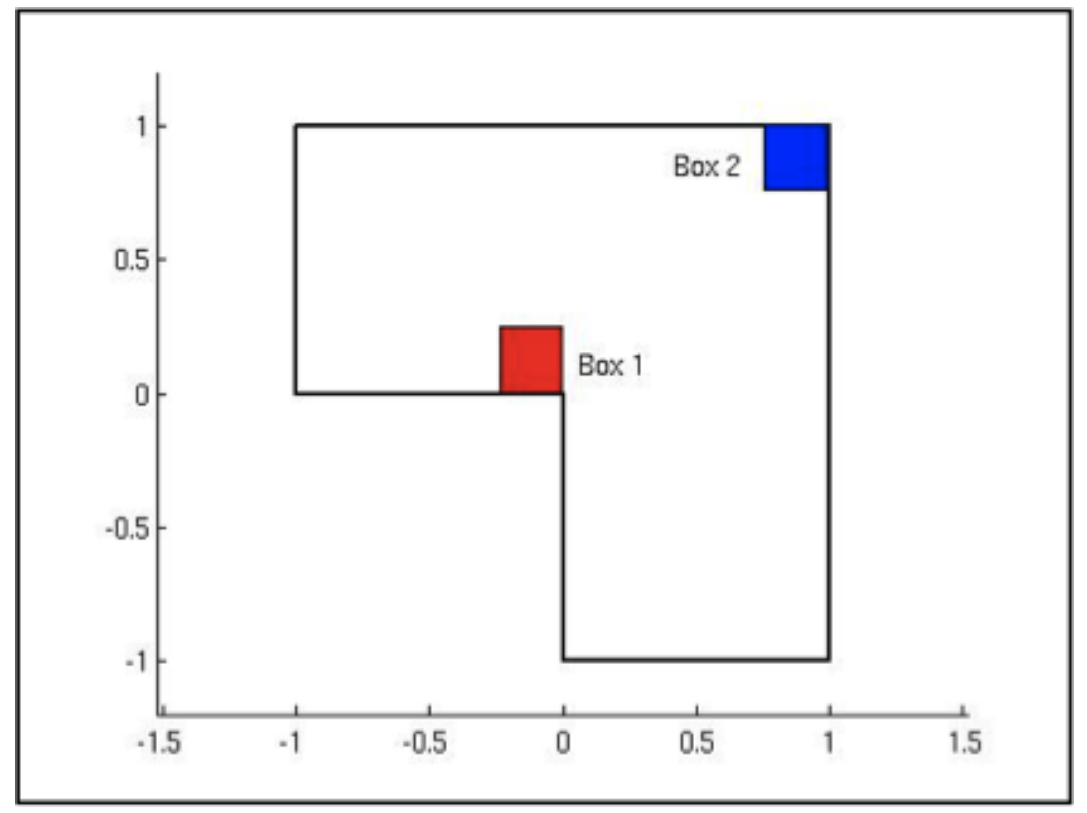

Figure 4: The regions used to average the temperatures for computing the race conditions

Table 3 provides the raw data for the race problem using both uniform and adaptive $h$-refinement. The results with the two methods are quite similar while the effective " $h$ " is the same. For the effective $h$ in the adaptive calculations we take the smallest mesh size as the meshes characteristic length for the purposes of verification. We find that this choice is consistent with the expectations of adaptive methods although it can be criticized on many technical grounds.

\subsubsection{Full data set Solution Verification Analysis}

This data set in particular will highlight the great impact that expert judgment can have on the fidelity of the analysis. This will not be so acutely felt in the analysis of the full data set, but will play a critical role when the data is limited and only available on a handful of coarse meshes. As we have seen previously, the expert judgment matters little when much data is available, but matters greatly when little data is present. We present two cases where the rate of convergence is constrained rather loosely $0.1 \leq$ $p \leq 2.0$, and the convergence results using robust verification are given in Table 4.

If we recognize that the rate of convergence will be quite low due to the 


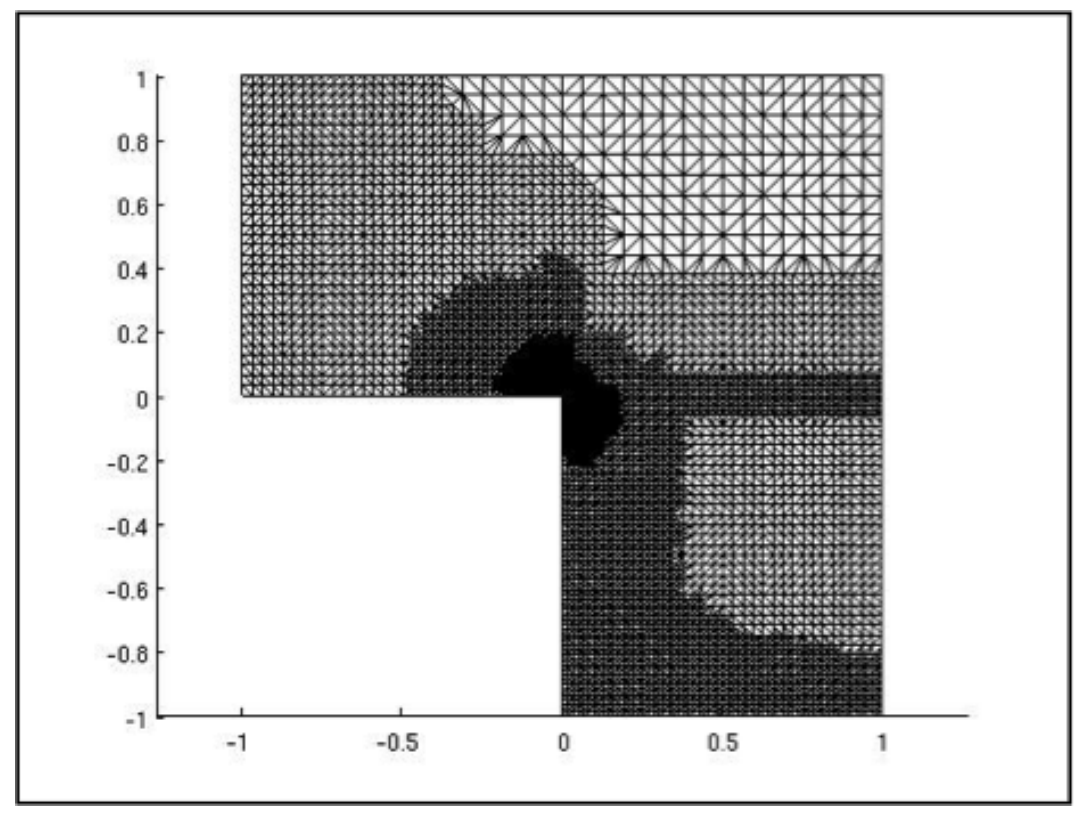

Figure 5: The adapted mesh from the race condition calculation

presence of significant singularities in the solution and change our expert judgment to a much lower convergence rate, $0.1 \leq p \leq 0.5$, the solution verification with robust verification changes significantly. The results of using this more pessimistic constraint is shown in Table 5 and reveals the nature of the impact of constraints. The impact of the constraints can also be clearly seen in Figure 6 where all the models are displayed. This is extremely evident with the constant mesh case that is data poor compared to the adaptive calculation.

The methodology provides a great deal of texture to the discussion of the relative merits of the solution methodology. Again, the results are selfconsistent; however, the convergence rate for the highly refined meshes available for the $h$-refinement is much lower than that for the relatively coarse meshes available for uniform refinement. Because of the low convergence rate the degree of improvement in the error estimate is quite low compared to the reduction in mesh size. The rate of convergence drops from approximately half for the coarse grids to a quarter for the highly refined meshes. We believe this reflects the true regularity of the solution and the measure examined. We note that the results are found for the integral average values in two regions. If these were point values the regularity would be lower 
Table 3: The numerical solutions of the thermal race problem time of equal temperatures using uniform and adaptive mesh solutions. The advantage of the adaptive mesh solution is readily apparent from the number of degrees of freedom required for very fine mesh resolution. The mesh scale "h" is the length of the finest elements in the mesh for the adaptive calculations and the standard element length in the standad calculations.

\begin{tabular}{lllll}
\hline " $h "$ & $\begin{array}{l}\text { Uniform } \\
\text { DOF }\end{array}$ & $\begin{array}{l}\text { Time of Equal } \\
\text { Time Difference }\end{array}$ & $\begin{array}{l}\text { Adaptive } \\
\text { DOF }\end{array}$ & $\begin{array}{l}\text { Time of Equal } \\
\text { Time Difference }\end{array}$ \\
\hline 0.12500 & 225 & 24.503 & 225 & 24.503 \\
0.06250 & 833 & 21.845 & 833 & 21.803 \\
0.03125 & 3201 & 19.874 & 2691 & 19.840 \\
0.015625 & 12545 & 18.429 & 5361 & 18.401 \\
0.0078125 & 49665 & 17.336 & 6329 & 17.312 \\
0.00390625 & 197633 & 16.484 & 7124 & 16.464 \\
0.001953125 & & & 7783 & 15.789 \\
0.0009765625 & & & 8401 & 15.241 \\
0.00048828125 & & & 8889 & 14.789 \\
0.000244140625 & & & 9318 & 14.413 \\
0.000122070313 & & & 9704 & 14.096 \\
0.000061035156 & & & 10053 & 13.828 \\
0.000030517578 & & & 10445 & 13.599 \\
0.000015258789 & & & 10697 & 13.403 \\
0.000007629395 & & & 10977 & 13.234 \\
0.000003814697 & & & 11250 & 13.089 \\
0.000001907349 & & & 11481 & 12.962 \\
0.000000953674 & & & 11685 & 12.853 \\
0.000000476837 & & & 11958 & 12.757 \\
0.000000238419 & & & 12089 & 12.674 \\
\hline
\end{tabular}

and the convergence rate would be lower still. Nonetheless the $h$-adaptivity lowers the error in the metric examined (time of equal temperatures) from $22 \%$ to over $4 \%$, which is a substantial reduction.

For the next three examples with limited data, the adaptivity provides little benefit on the coarse meshes. As a result only a single solution verification result is shown. 
Table 4: The verification analysis numerical solutions of the thermal race problem time of equal temperatures using uniform and adaptive mesh solutions for the loose constraint, $0.1 \leq p \leq 2.0$.

\begin{tabular}{lll}
\hline & Uniform & h-adaptive \\
\hline Converged Value & 14.7133 & 12.3533 \\
RoC & 0.5391 & 0.2518 \\
UQ of Value & \pm 3.2865 & \pm 0.5780 \\
$F_{s}$ & 2.856 & 2.802 \\
UQ of RoC & \pm 0.6439 & \pm 0.1419 \\
UQ of Value with Bias & -2.9885 & -0.0992 \\
& +3.4851 & +0.7040 \\
UQ of RoC with Bias & -0.4658 & -0.5203 \\
& +1.3835 & +0.7367 \\
\hline
\end{tabular}

Table 5: The verification analysis numerical solutions of the thermal race problem time of equal temperatures using uniform and adaptive mesh solutions for the tight constraint, $0.1 \leq p \leq 0.5$.

\begin{tabular}{lll}
\hline & Uniform & h-adaptive \\
\hline Converged Value & 14.0974 & 12.2555 \\
RoC & 0.4411 & 0.2335 \\
UQ of Value & \pm 0.9891 & \pm 0.2409 \\
$F_{s}$ & 1.414 & 1.576 \\
UQ of RoC & \pm 0.1087 & \pm 0.0615 \\
UQ of Value with Bias & -1.1409 & -0.3025 \\
& +0.9690 & +0.1798 \\
UQ of RoC with Bias & -0.1155 & -0.0615 \\
& +0.1067 & +0.050079 \\
\hline
\end{tabular}

\subsubsection{Two Point Calculation Solution Verification Analysis}

Again, we will highlight the impact of the expert judgment on the analysis when available. With well-chosen constraints extremely good results can be achieved. With the well-chosen, tight constraint, $0.1 \leq p \leq 0.5$, that acknowledges the expectation of low convergence rates we get an estimate of 

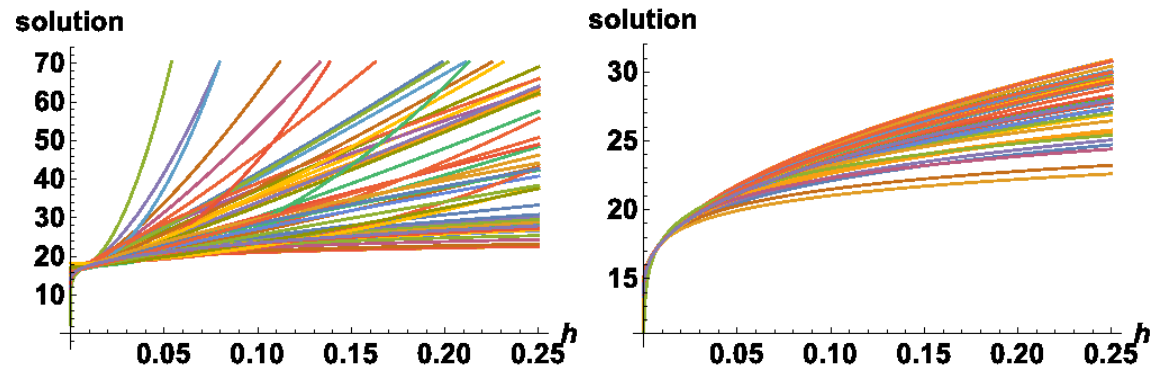

Figure 6: The sets of 19 models for the full set of non-adaptive solutions for the thermal race problem. The left plot shows the relatively loose constraint on the convergence rate, $0.1 \leq p \leq 2.0$, and the right plot shows the tigther constraint, $0.1 \leq p \leq 0.5$. The tighter constraint produces far smaller error and a more accurate estimate of the converged solution.

mesh converged solution, $\Delta t_{k}=5.056 \pm 13.493+C h_{k}^{0.212 \pm 0.126}, F_{s}=1.804$ (note the QoI is a time difference). With a more optimistic (higher) constraint on the convergence rate we estimate, $0.1 \leq p \leq 2.0, \Delta t_{k}=20.367 \pm$ $1.271+C h_{k}^{1.572 \pm 0.783}, F_{s}=1.860$. This is clearly a poor estimate in virtually every respect. This shows the impact and power of properly chosen expert judgment. Clearly broadly chosen constraints on the convergence rate produce broad error estimates. In this case the standard GCI estimate of numerical error gives $\Delta t=7.974$, which does not bound the mesh converged solution given by the adaptive mesh method. The difference in the quality of the estimates can be seen in Figure 7.

\subsubsection{Three Point Calculation Solution Verification Analysis}

Here we just use the lower estimate of rate of convergence to constrain the optimizations in our solution verification procedure. Using this constraint on three data points gives (38 models computed), $\Delta t_{k}=11.906 \pm 9.493+$ $C h_{k}^{0.345 \pm 0.266}, F_{s}=2.191$. The standard least squares regression fit gives, $\Delta t_{k}=14.202+25.213 h_{k}^{0.430}$ and a numerical uncertainty of 17.015. Stern's gives a smaller estimate and Stern and Xing's procedure gives a much larger error estimates with a theoretical convergence rate set to $p_{t h}=0.25$.

\subsubsection{Four Point Calculation Solution Verification Analysis}

Again we just use the lower estimate of rate of convergence to constrain the optimizations in our solution verification procedure. Using this constraint on three data points gives (38 models computed), $\Delta t_{k}=14.339 \pm$ $1.956+C h_{k}^{0.438 \pm 0.133}, F_{s}=1.478$. This solution provides much smaller uncer- 

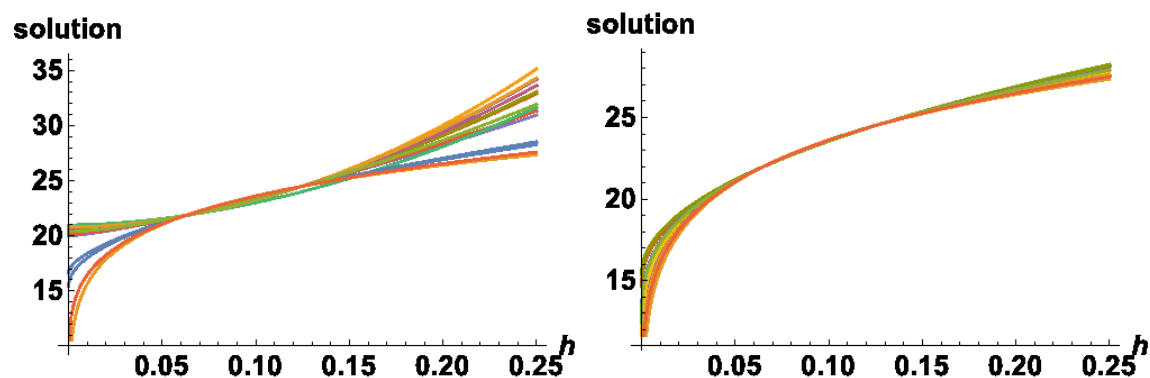

Figure 7: The sets of 19 models for the two coarse step size solutions for the thermal race problem. The left plot shows the relatively loose constraint on the convergence rate, $0.1 \leq p \leq 2.0$, and the right plot shows the tigther constraint, $0.1 \leq p \leq 0.5$. The tighter constraint produces far smaller error and a more accurate estimate of the converged solution.

tainties than the three data point analysis. At this point the error estimates are substantially less than the standard GCI approach gives or its alternatives. The usual least squares regression fit gives, $\Delta t_{k}=14.339+25.272 h_{k}^{0.434}$ and a numerical uncertainty of 12.271. Stern's gives a smaller estimate and Stern and Xing's procedure gives a much larger error estimates with a theoretical convergence rate set to $p_{t h}=0.25$.

\subsection{Simple Neutronics}

We will finish the demonstrations by examining the verification methodology on a more complex error model using two discrete variables. In this case we will examine the six-dimensional solution of radiation transport and study verification in a single space discretization (three dimensions) and the angular discretization (for two dimensions), the final dimension of energy will not be examined, thus held fixed for all computations. Denovo [8] is a discrete ordinates radiation transport method used to solve the Boltzmann transport equation (time-independent),

$$
\begin{aligned}
& \Omega \cdot \nabla \phi(x, \Omega, E)+\sigma(x, E) \phi(x, \Omega, E)= \\
& \int d E^{\prime} \int_{4 \pi} d \Omega^{\prime} \sigma_{s}\left(x, \Omega^{\prime} \cdot \Omega, E^{\prime} \rightarrow E\right) \phi\left(x, \Omega^{\prime}, E^{\prime}\right) \\
& \frac{\xi(E)}{4 \pi k} \int d E^{\prime} \int_{4 \pi} d \Omega^{\prime} \nu \sigma_{f}\left(x, \Omega^{\prime} \cdot \Omega, E^{\prime} \rightarrow E\right) \phi\left(x, \Omega^{\prime}, E^{\prime}\right)
\end{aligned}
$$

describing the behavior of neutron populations in space. The methodology used by Denovo is referred to as deterministic transport to distinguish it from 
statistical methods based on the Monte Carlo method, although Denovo does contain the capacity to compute Monte Carlo solutions. The code contains the capability to use several different spatial approximations to the streaming term, $\Omega \cdot \nabla \phi(x, \Omega, E)$ in Equation (22) as well as different quadrature formula for the integral terms.

The spatial approximation to the first-order streaming term includes the step characteristic method (i.e., donor cell or upwind in the parlance of hydrodynamics methods), and linear discontinuous (i.e., discontinuous Galerkin), trilinear discontinuous, and the classical diamond differencing with negative flux fix-up. These methods each carry certain advantages and have been examined extensively in the literature $[11,12]$. The spatial grid can be 1-, 2-, or 3-dimensional and non-uniform in nature. For our study here the mesh will be uniform in all three dimensions.

The angular discretization includes the classical level symmetric method, which is the default. The order of the quadrature can take even values from 2 to 24, for the level-symmetric quadrature the points are visualized in Figure 8. Other available quadratures are Gauss-Legendre, Quadruple Range, Galerkin, and a linear discontinuous finite element (LDFE) approach. We can examine these quadratures with respect to mesh convergence in the angular variable although expectations for the rate of convergence are sparse in the literature. In this work we will focus on the level-symmetric quadrature. Denovo employs the Koch-Baker-Alcouffe sweep method for solution along with accelerated source iteration. The code can compute in parallel.

We have examined a relatively simple problem used in the Denovo testing suite. The energy group structure is not modified although, as noted, this would be an excellent future study. The problems used are focused upon separate physical effects, either upscatter (scattering where the neutron moves to a higher energy), or downscatter (where the neutron moves to lower energy). The upscatter problem uses five energy groups, and has an isotropic source. An example of the type of solution seen for this problem is shown in Figure 9.

In order to apply verification to neutronics we must treat the angular variable. The angular quadrature is defined by the order of the quadrature and here our attention is primarily directed toward the level symmetric set used as the default in Denovo. This method is known as the "Sn" method where " $n$ " is the order of the quadrature, and the number of quadrature points is given by $n(n+2) / 2$, which scales as $n^{2}$. The angular space is two dimensional; thus, the analog to $h$ in space is $1 / n$ in angle. This is similar to the ansatz made by Jarrell in his thesis [10]. We then take the angular 

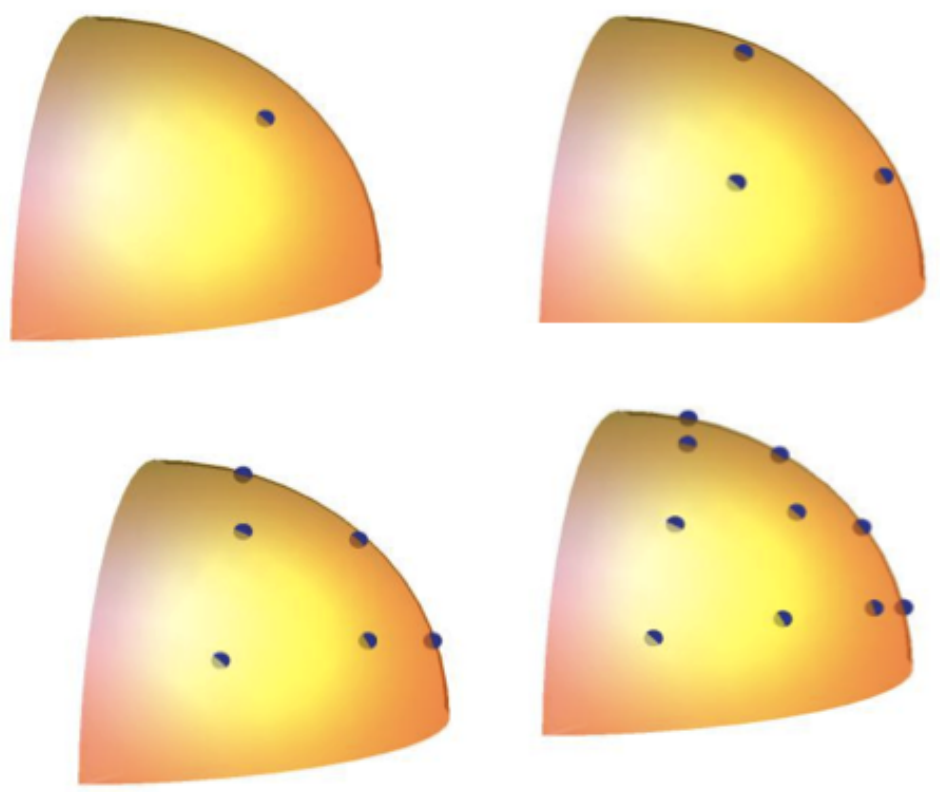

Figure 8: Example of the placement of quadrature points for the level symmetric quadrature for the S2, S4, S6 and S8. A single octant of the solid sphere is displayed with the location of the integration points.

error to be

$$
A_{k}=\tilde{A}+C_{n} / n^{p_{n}}
$$

and the coupled angular-space equation with discretization error to be

$$
A_{k}=\tilde{A}+C_{h} h^{p_{h}}+C_{n} / n^{p_{n}}
$$

or

$$
A_{k}=\tilde{A}+C_{h} h^{p_{h}}+C_{n} / n^{p_{n}}+C_{h n}\left(\frac{h}{n}\right)^{p_{h n}},
$$

These are the specific forms that we examine from the set of calculations conducted with Denovo. We have tested Equation (24) in comparison to Equation (25) and found that the simpler form of the error model performs better as measured by the requisite uncertainty. Some general conclusions regarding the propriety of these forms will be one of the preliminary conclusions from this study. 

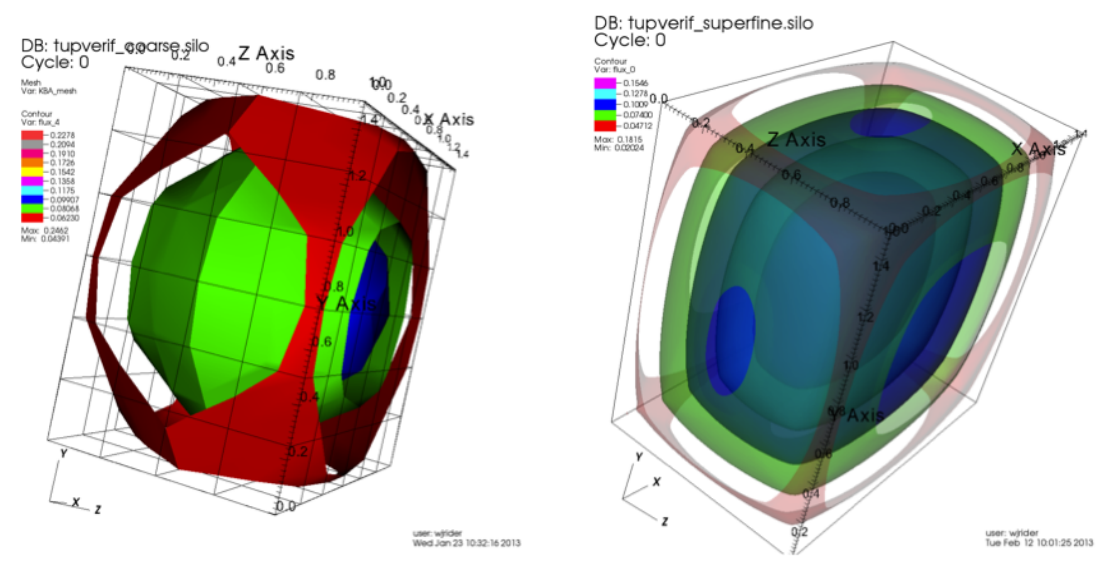

Figure 9: A contour plot of the integrated scalar flux for the energy group 5 on the finest and coarsest meshes for 5 different values of the flux. It is a smooth isotropic problem as shown, which should give relatively well-behaved results in the verification study. Sn6 is used for the angular integration.

The basic results for the upscatter problem are summarized in Table 6 for the different spatial and angular "meshes" used. These results will then be analyzed with regard to the first separate spatial and angular convergence, and then again for the coupled error. The results indicate to the casual observer that the solution is quite well "mesh-resolved" in both space and angle for the finest "meshes" chosen.

\subsubsection{Full data set analysis}

The analysis using the new verification methodology is provided in Tab. 7 . This analysis used constraints on the convergence rates, $1 \leq p_{h} \leq 5$ and $1 \leq p_{n} \leq 4$ with $\tilde{A} \geq 0$ with theoretically expected rates of $p_{h, t h}=3$ and $p_{n, t h}=2$. Given the median statistics used in our methodology all of the results for converged fluxes and convergence rates are consistent well within the $95 \%$ stated confidence interval. It has been noted that this problem is not useful for measuring spatial convergence because the approximation becomes semi-analytical. This accounts for high rate of convergence for the spatial differencing and the lack of distinction between the step and LD methods. Our proposed error form for the angular quadrature appears to hold up to the examination as confirmed by the approximately second-order convergence for the level-symmetric approach. Figures 10 and 11 provide more texture to the results showing the results of the models solved given the above data. The spatial error is better behaved than the angular error, 

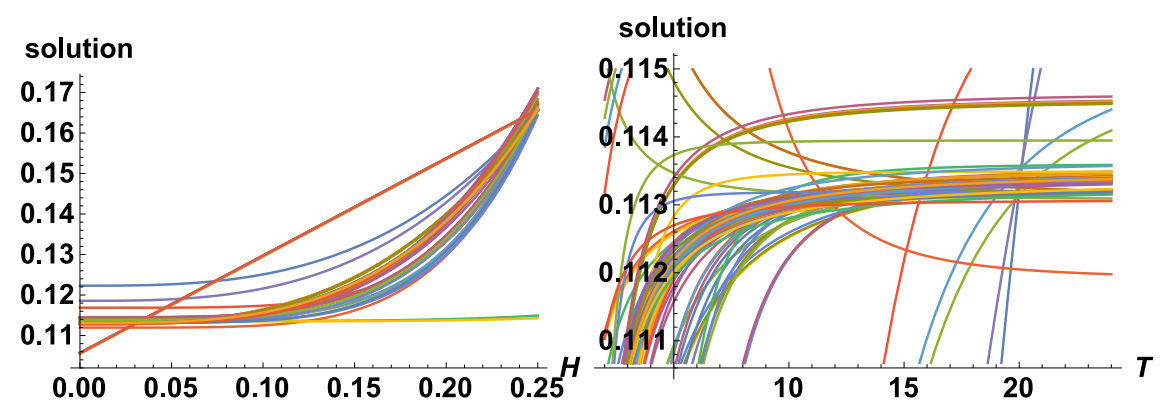

Figure 10: Ensemble of models for space (left, $n=24$ ) and angular (right, $h=0.05$ ) error for the coupled space-angle convergence analysis. Both sets of discretizations appear to be well behaved despite a handful of outliers (which are removed by the application of the median statistics).

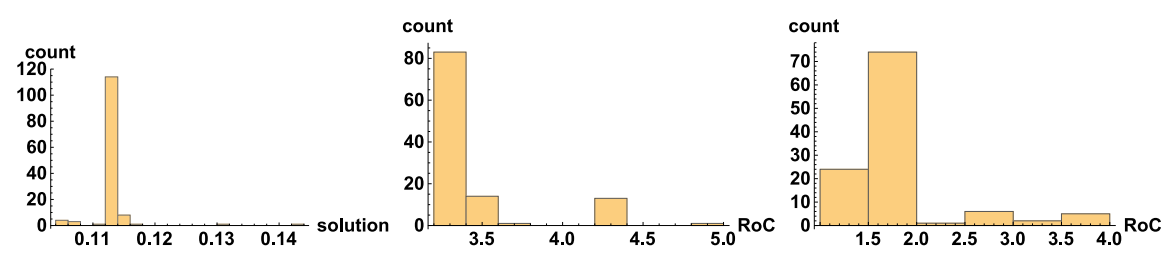

Figure 11: PDFs of the converged solution (left), spatial convergence rate (center) and angular convergence rate (right).

but both are reasonable. The effective probability distribution functions from the analysis are vivid reminders of the non-Gaussian nature and nonsymmetric of the analysis.

The nature of the median statistics removes irregular results that can result from the roughness of the data or unreliability of the optimization solution. The other primary sources of difficulty are the results obtained with the coarsest angular quadrature, $\mathrm{Sn} 2$, which produces results and convergence uncharacteristic of the refined quadratures. We note, again, that the optimization or regression can sometimes fail spectacularly, and these results must be discarded to avoid corrupting results. The median statistics readily accomplish this.

\subsubsection{Two Calculation Analysis}

To highlight the capability for the robust verification analysis of coarsely resolved solutions with insufficient data we will provide the results from two data points. We note that the two data points are used to determine five 
constants in the error equation (24). The data points use different spatial mesh spacing and quadratures, $[(0.25,2,0.16124),(0.125,4,0.11685)]$ for the LD spatial discretization. In the robust verification procedure we produce 19 models that are scrutinized by the robust statistics. This produces the following result to be compared $A_{k}=0.103324 \pm 0.025048+C_{h} h_{k}^{3.34978 \pm 1.31163+}$ $C_{n} / n_{k}^{2.10524 \pm 1.91166}$. We believe that these results are extremely good considering the sparsity of the data, and the coarseness of the discretization. In this case the safety factor that the algorithm produces, $F_{s}=2.85$, is nearly the same as the standard $F_{s}=3$.

\section{Conclusion and Prospectus}

Our methodology represents a substantial change in the approach to conducting the detailed verification analysis of calculations. Our intent is to remove some degree of a priori assumptions and make the overall conduct of verification more self-contained. Our procedure is designed to make the role of expert judgment evident and transparent. The magnitude of the error estimated is determined by the data itself and expert judgment regarding the numerical method's and the problem's characteristics. Our approach has been tested on a diverse set of calculations from differing sources, demonstrating their capacity to reasonably compute numerical error estimates. In addition, the results of the analysis reveal more information about the structure of the error including uncertainties on the rates of convergence. We have provided several demonstrations including a code verification result. In the future we anticipate using this discretization error analysis methodology on data sets from a wider variety of calculations of interest.

\section{Acknowledgment}

The authors would like to thank Tim Trucano, Vince Mousseau, Tom Evans, Doug Kothe, Patrick Knupp, Bill Oberkampf, Greg Weirs, and Rich Hills for numerous discussions and informative suggestions. The DOE ASC $\mathrm{V} \& \mathrm{~V}$ program has provided support for the development of the robust verification methodology. The DOE CASL program and the Sandia National Laboratories, LDRD program, have provided further support for the verification of calculations.

Sandia National Laboratories is a multi-program laboratory managed and operated by Sandia Corporation, a wholly owned subsidiary of Lockheed Martin Corporation, for the U.S. Department of Energys National Nuclear Security Administration under contract DE-AC04-94AL85000. This 
work was performed under the auspices of the United States Department of Energy by Los Alamos National Security, LLC, at Los Alamos National Laboratory under contract DE-AC52-06NA25396.

\section{References}

[1] BAbušKa, L., And Suri, M. The optimal convergence rate of the p-version of the finite element method. SIAM Journal on Numerical Analysis 24, 4 (1987), 750-776.

[2] Banks, J. W., Aslam, T., And Rider, W. On sub-linear convergence for linearly degenerate waves in capturing schemes. Journal of Computational Physics 227, 14 (2008), 6985-7002.

[3] BJork, A. Numerical Methods for Least Squares Problems. SIAM, 1996.

[4] Donoho, D. L., And Huber, P. J. The notion of breakdown point. A Festschrift for Erich L. Lehmann 157184 (1983).

[5] EÇA, L., And Hoekstra, M. A procedure for the estimation of the numerical uncertainty of cfd calculations based on grid refinement studies. Journal of Computational Physics 262 (2014), 104-130.

[6] EÇA, L., And Hoekstra, M. Discretization uncertainty estimation based on a least squares version of the grid convergence index. In Proceedings of the Second Workshop on CFD Uncertainty Analysis (Oct. 2006).

[7] EÇA, L., VAz, G., And Hoekstra, M. Code verification, solution verification and validation in rans solvers. In ASME 2010 29th International Conference on Ocean, Offshore and Arctic Engineering (2010), American Society of Mechanical Engineers, pp. 597-605.

[8] Evans, T. Denovo: A radiation transport code for nuclear applications. Tech. rep., Oak Ridge National Laboratory, 2010. SciDAC SciADS 2010, Snowbird Utah, ORNL.

[9] Huber, P. Robust Statistical Procedures. SIAM, 1996.

[10] Jarrell, J. An Adaptive Angular Discretization Method For NeutralParticle Transport in Three-Dimensional Geometries. $\mathrm{PhD}$ thesis, Texas A\&M University, 2010. 
[11] Larsen, E., And Nelson, P. Finite-difference approximations and superconvergence for the discrete-ordinate equations in slab geometry. SIAM Journal on Numerical Analysis 19, 2 (1982), 334-348.

[12] Lewis, E. E., ANd Miller, W. P. Numerical Methods for Neutron Transport. Wiley Interscience, 1984.

[13] Leys, C., Klein, O., Bernard, P., and Licata, L. Detecting outliers: Do not use standard deviation around the mean, use absolute deviation around the median. Journal of Experimental Social Psychology 49, 4 (2013), 764-766.

[14] Lin, P. T., Sala, M., Shadid, J. N., and Tuminaro, R. S. Performance of fully-coupled algebraic multilevel domain decomposition preconditioners for incompressible flow and transport. nt. J. Num. Meth. Eng 67 (2006), 208-225.

[15] Majda, A., AND Osher, S. Propogation of error into regions of smoothness for accuracte difference approximations to hyperbolic equations. Communications on Pure and Applied Mathematics 30 (1977), $671-705$.

[16] Oberkampf, W. L. Roy, C. J. Verification and Validation in Scientific Computing. Cambridge University Press, 2010.

[17] Pawlowski, R. P., Shadid, J. N., Simonis, J. P., And Walker, H. F. Globalization techniques for newton-krylov methods and applications to the fully coupled solution of the navier-stokes equations. SIAM Review 48(4) (2006), 700-721.

[18] Rider, W. J., And Kamm, J. R. Advanced Solution Verification of CFD Solutions for LES of Relevance to GTRF Estimates, SAND20127199P. Tech. rep., Sandia National Laboratories, 2012.

[19] RoAche, P. Verification and Validation in Computational Science and Engineering. Hermosa Publishers, 1998.

[20] Roache, P. Fundamentals of Verification and Validation. Hermosa Publishers, 2009.

[21] Rodriguez, S. B., ANd El-Genk, M. S. Numerical investigation of potential elimination of hot streaking" and stratification in the vhtr lower plenum using helicoid inserts. Nuclear Engineering and Design 240 (2010), 99-1004. 
[22] Rousseeuw, P. J., And Croux, C. Alternatives to the median absolute deviation. Journal of the American Statistical association 88, 424 (1993), 1273-1283.

[23] Smagorinsky, J. The beginnings of numerical weather prediction and general circulation modeling: Early recollections. Advances in Geophysics 25 (1983), 3-37.

[24] Stern, F., Wilson, R. V., Coleman, H. W., and Paterson, E. G. Comprehensive approach to verification and validation of $\mathrm{cfd}$ simulationspart 1: Methodology and procedures. J. Fluids Engrng. 123 (2001), 793802.

[25] Xing, T., And Stern, F. Factors of safety for richardson extrapolation. J. Fluids Engrng. 132 (2010), 1-13. 
Table 6: The raw results for the integrated scalar flux from the upscatter problem are shown for space-angle discretizations

\begin{tabular}{llll}
\hline$h(\Delta x)$ & $n$ & LD int. flux & Step int. flux \\
\hline 0.25000 & 2 & 0.16124 & 0.14930 \\
0.12500 & 2 & 0.11277 & 0.11017 \\
0.06250 & 2 & 0.10791 & 0.10684 \\
0.03125 & 2 & 0.10754 & 0.10703 \\
0.01563 & 2 & 0.10752 & \\
0.25000 & 4 & 0.16398 & 0.15387 \\
0.12500 & 4 & 0.11685 & 0.11423 \\
0.06250 & 4 & 0.11188 & 0.11069 \\
0.03125 & 4 & 0.11148 & 0.11087 \\
0.01563 & 4 & 0.11145 & \\
0.25000 & 6 & 0.16477 & 0.15555 \\
0.12500 & 6 & 0.11762 & 0.11541 \\
0.06250 & 6 & 0.11268 & 0.11170 \\
0.03125 & 6 & 0.11227 & 0.11177 \\
0.01563 & 6 & 0.11224 & \\
0.25000 & 8 & 0.16517 & 0.15599 \\
0.12500 & 8 & 0.11800 & 0.11581 \\
0.06250 & 8 & 0.11307 & 0.11209 \\
0.03125 & 8 & 0.11266 & 0.11216 \\
0.01563 & 8 & 0.11262 & \\
0.25000 & 12 & 0.16549 & 0.15647 \\
0.12500 & 12 & 0.11829 & 0.11619 \\
0.06250 & 12 & 0.11336 & 0.11243 \\
0.03125 & 12 & 0.11295 & 0.11247 \\
0.01563 & 12 & 0.11292 & \\
0.25000 & 16 & 0.16563 & 0.15670 \\
0.12500 & 16 & 0.11841 & 0.11636 \\
0.06250 & 16 & 0.11348 & 0.11258 \\
0.03125 & 16 & 0.11307 & 0.11261 \\
0.01563 & 16 & 0.11304 & \\
0.25000 & 20 & 0.16570 & \\
0.12500 & 20 & 0.11848 & \\
0.06250 & 20 & 0.11355 & \\
0.03125 & 20 & 0.11314 & \\
0.01563 & 20 & 0.11311 & \\
0.25000 & 24 & 0.16575 & 0.15689 \\
0.12500 & 24 & 0.11858 & 0.11651 \\
0.06250 & 24 & 0.11359 & 0.11271 \\
0.03125 & 24 & 0.11318 & 0.11273 \\
0.01563 & 24 & 0.11315 & \\
\hline & & &
\end{tabular}


Table 7: Convergence results for the downscatter problem, the step method is examined with coupled analysis, while the LD (linear discontinuous) is examined for space and angle alone as well as coupled space-angle for the convergence. We note that the smallest uncertainties are achieved with the coupled method. This problem is noted as being inapplicable to analyzing the spatial operators because of the nature of the transport rendering the step method to be uncharacteristically accurate.

\begin{tabular}{lllllll}
\hline Case & $\begin{array}{l}\text { Converged } \\
\text { Flux }\end{array}$ & \pm flux & $\begin{array}{l}\text { Spatial } \\
\text { rate, } p_{h}\end{array}$ & & $\begin{array}{l}\text { Angular } \\
\text { rate, } p_{n}\end{array}$ & \pm rate \\
\hline Step space-angle & 0.112767 & 0.000212 & 3.57327 & 0.13475 & 1.80497 & 0.85473 \\
LD space & 0.113143 & 0.000126 & 3.66037 & 1.05584 & & \\
LD angle & 0.113615 & 0.000081 & & & 2.23472 & 0.70415 \\
LD space-angle & 0.113222 & 0.000141 & 3.28949 & 0.04058 & 1.62063 & 0.54076 \\
\hline
\end{tabular}

